\title{
Wavefront analysis from its slope data
}

\section{Virendra N. Mahajan, Eva Acosta}

Virendra N. Mahajan, Eva Acosta, "Wavefront analysis from its slope data," Proc. SPIE 10375, Current Developments in Lens Design and Optical

Engineering XVIII, 103750A (30 August 2017); doi: 10.1117/12.2282995

SPIE Event: SPIE Optical Engineering + Applications, 2017, San Diego, California, United States 


\title{
Wavefront analysis from its slope data
}

\author{
Virendra N. Mahajan* and Eva Acosta** \\ *College of Optical Sciences, University of Arizona, Tucson, AZ 85721 \\ **Area de Optica, Departmento de Fisica Aplicada, Universidade de Santiago de Compostela, Galicia, Spain \\ Corresponding author: Virendra N. Mahajan <virmahajan12@gmail.com>
}

\begin{abstract}
In the aberration analysis of a wavefront over a certain domain, the polynomials that are orthogonal over and represent balanced wave aberrations for this domain are used. For example, Zernike circle polynomials are used for the analysis of a circular wavefront. Similarly, the annular polynomials are used to analyze the annular wavefronts for systems with annular pupils, as in a rotationally symmetric two-mirror system, such as the Hubble space telescope. However, when the data available for analysis are the slopes of a wavefront, as, for example, in a ShackHartmann sensor, we can integrate the slope data to obtain the wavefront data, and then use the orthogonal polynomials to obtain the aberration coefficients. An alternative is to find vector functions that are orthogonal to the gradients of the wavefront polynomials, and obtain the aberration coefficients directly as the inner products of these functions with the slope data. In this paper, we show that an infinite number of vector functions can be obtained in this manner. We show further that the vector functions that are irrotational are unique and propagate minimum uncorrelated additive random noise from the slope data to the aberration coefficients.
\end{abstract}

Keywords: Wavefront analysis, wavefront slope data, circular wavefront, annular wavefront, optical testing.

\section{INTRODUCTION}

For an optical system with a circular pupil, the aberration coefficients of its circular wavefront can be obtained by expanding it in terms of Zernike circle polynomials that are orthonormal over the pupil and represent balanced classical aberrations for such a pupil [1]. However, if the measured data are the 2D slopes or the gradient of a wavefront, as, for example, in a Shack-Hartmann sensor, then we can either integrate the slope data to obtain the wavefront data and then use the Zernike polynomials to obtain the Zernike coefficients. There is another two-step approach to obtain these coefficients. The slope data is expanded in terms of the gradients of the balanced wave aberrations that yield minimum variance of the transverse ray aberrations $[1,2\}$, and the coefficients thus obtained are transformed to obtain the Zernike coefficients. These gradient polynomials are the same as those obtained by orthogonalizing the gradients of Zernike polynomials [3\}.

An alternative is to find vector functions that are orthogonal to the gradients of Zernike polynomials and whose inner products with the slope data yield the Zernike coefficients directly [4]. We show that an infinite number of vector functions can be obtained in this manner. However, if we obtain these vector functions as the gradient of a scalar function so that they are irrotational, then they are unique and propagate minimum uncorrelated additive random noise from the slope data to the Zernike coefficients.

We start this paper by defining the mathematical problem in Section 2, and illustrate in Section 3 with simple examples an infinite number of vector functions that can be used to obtain the aberration coefficients. In Section 4, we show that irrotational vector functions propagate minimum noise from the slope data to the aberration coefficients [5,6]. Accordingly, we obtain them in Section 5 as the gradient of a scalar function. We find that these 
functions are polynomials for a circular wavefront [7], but general functions for an annular wavefront, as discussed very briefly in Section 6. [8]. The paper ends in Section 7 with brief discussion and conclusions.

\section{ANALYSIS OF A CIRCULAR WAVEFRONT}

A circular wavefront represented by an aberration function $W(\rho, \theta)$ can be expanded in terms of $J$ Zernike circle polynomials $Z_{j}(\rho, \theta)$ in the form [1]

$$
W(\rho, \theta)=\sum_{j=1}^{J} a_{j} Z_{j}(\rho, \theta)
$$

where $a_{j}$ is the Zernike coefficient of the polynomial $Z_{j}$. Multiplying both sides of Eq. (1) by $Z_{j}(\rho, \theta)$, integrating over the unit pupil, and using the orthonormality of the Zernike polynomials, we obtain the Zernike coefficients:

$$
a_{j}=\frac{1}{\pi} \int_{0}^{1} \int_{0}^{2 \pi} W(\rho, \theta) Z_{j}(\rho, \theta) \rho d \rho d \theta
$$

We want to find vector functions $\vec{G}_{j}(\rho, \theta)$ that yield the aberration coefficients from the wavefront slope data $\nabla W(\rho, \theta)$ according to

$$
a_{j}=\frac{1}{\pi} \int_{0}^{1} \int_{0}^{2 \pi} \vec{G}_{j}(\rho, \theta) \cdot \nabla W(\rho, \theta) \rho d \rho d \theta
$$

Substituting for $\nabla W$ from Eq. (1) into Eq. (3), we obtain

$$
a_{j}=\frac{1}{\pi} \int_{0}^{1} \int_{0}^{2 \pi} \vec{G}_{j}(\rho, \theta) \cdot \sum_{j=1}^{J} a_{j} \nabla Z_{j}(\rho, \theta) \rho d \rho d \theta
$$

showing that the vector functions must be orthogonal to the gradients of Zernike polynomials.

To find the vector functions, we start with the identity

$$
\nabla \cdot(W \vec{G})=W(\nabla \cdot \vec{G})+\vec{G} \cdot(\nabla W)
$$

Integrating both sides over a unit pupil, we may write

$$
\int_{0}^{1} \int_{0}^{2 \pi} \nabla \cdot(W \vec{G}) \rho d \rho d \theta=\int_{0}^{1} \int_{0}^{2 \pi} W(\nabla \cdot \vec{G}) \rho d \rho d \theta+\int_{0}^{1} \int_{0}^{2 \pi} \vec{G} \cdot(\nabla W) \rho d \rho d \theta
$$

From Green's theorem [9], the left-hand side integral in Eq. (9) can also be written in terms of the line integral about the contour of the pupil, i.e.,

$$
\int_{0}^{1} \int_{0}^{2 \pi} \nabla \cdot(W \vec{G}) \rho d \rho d \theta=\underset{1}{\oint} W(1, \theta) \vec{G}(1, \theta) \cdot d \vec{l}
$$

where $d \vec{l}$ is a differential contour vector element pointing out of the circumference of the unit pupil. From Eqs. (6) and (7), we may write

$$
\int_{0}^{1} \int_{0}^{2 \pi} W(\rho, \theta) \nabla \cdot \vec{G}_{j}(\rho, \theta) \rho d \rho d \theta=-\int_{0}^{1} \int_{0}^{2 \pi} \nabla W(\rho, \theta) \cdot \vec{G}_{j}(\rho, \theta) \rho d \rho d \theta+\oint_{1}^{\oint} W(1, \theta) \vec{G}(1, \theta) \cdot d \vec{l} .
$$


If we can find a vector function $\vec{G}_{j}(\rho, \theta)$ such that its divergence $\nabla \cdot \vec{G}_{j}$ is given by

$$
\nabla \cdot \vec{G}_{j}=-Z_{j}(\rho, \theta)
$$

and satisfies the boundary condition

$$
\vec{G}_{j}(1, \theta) \cdot d \vec{l}=0,
$$

so that the line integral in Eq. (8) about the circumference of the unit pupil vanishes, then this equation reduces to Eq. (3). Thus, we want to find vector functions that satisfy Eq. (9) subject to the boundary condition of Eq. (10).)

\section{INFINITE NUMBER OF VECTOR FUNCTIONS}

Now, we consider simple examples of Zernike polynomials and illustrate that there is an infinite set of vector functions that can be constructed to obtain the Zernike coefficients [10]. In polar coordinates, Eq. (9) can be written

$$
\nabla \cdot \vec{G}_{j}(\rho, \theta)=\frac{1}{\rho} \frac{\partial}{\partial \rho}\left(\rho G_{j \rho}\right)+\frac{1}{\rho} \frac{\partial}{\partial \theta} G_{j \theta}=-Z_{j}(\rho, \theta)
$$

where $G_{j \rho}$ and $G_{j \theta}$ are the radial and angular components of the $\vec{G}_{j}$ according to

$$
\vec{G}_{j}(\rho, \theta)=G_{j \rho}(\rho, \theta) \hat{\rho}+G_{j \theta}(\rho, \theta) \hat{\theta} .
$$

Here, $\hat{\rho}$ and $\hat{\theta}$ are unit vectors in the polar coordinates. They are related to the corresponding Cartesian unit vectors $\hat{i}$ and $\hat{j}$ according to

$$
\hat{\rho}=\cos \theta \hat{i}+\sin \theta \hat{j}
$$

and

$$
\hat{\theta}=-\sin \theta \hat{i}+\cos \theta \hat{j}
$$

\subsection{Wavefront Tilt}

First we consider the Zernike polynomial representing wavefront tilt:

$$
Z_{2}(\rho, \theta)=\rho \cos \theta
$$

where we focus on its form, and ignore any normalization constants. The full form of a Zernike polynomial for primary aberrations is given in Table 1 . Consider the corresponding vector function $\vec{G}_{2}(\rho, \theta)$ for which $G_{2 \theta}=0$. Thus we may write

$$
\frac{1}{\rho} \frac{\partial}{\partial \rho}\left(\rho G_{2 \rho}\right)=\rho \cos \theta
$$

which yields

$$
G_{2 \rho}=\frac{1}{3} \rho^{2} \cos \theta+f(\theta),
$$

where $f(\theta)$ is an arbitrary function of $\theta$ that we choose to be $-(1 / 3) \cos \theta$ so that $\vec{G}_{2}(\rho, \theta)$ satisfies the boundary 
Table 1. Orthonormal Zernike circle polynomials $Z_{j}(\rho, \theta)$. The indices $j, n$, and $m$ are called the polynomial number, radial degree, and azimuthal frequency, respectively. The polynomials $\boldsymbol{Z}_{j}$ are ordered such that an even $j$ corresponds to a symmetric polynomial varying as $\cos m \theta$, while an odd $j$ corresponds to an antisymmetric polynomial varying as $\sin m \theta$. A polynomial with a lower value of $n$ is ordered first, and for a given value of $n$, a polynomial with a lower value of $m$ is ordered first.

\begin{tabular}{lllll}
\hline$j$ & $n$ & $m$ & $Z_{j}(\rho, \theta)$ & Aberration \\
\hline 2 & 1 & 1 & $2 \rho \cos \theta$ & $x$-tilt \\
3 & 1 & 1 & $2 \rho \sin \theta$ & $y$-tilt \\
4 & 2 & 0 & $\sqrt{3}\left(2 \rho^{2}-1\right)$ & Defocus \\
5 & 2 & 2 & $\sqrt{6} \rho^{2} \sin 2 \theta$ & $45^{\circ}$ Primary astigmatism \\
6 & 2 & 2 & $\sqrt{6} \rho^{2} \cos 2 \theta$ & $0^{\circ}$ Primary astigmatism \\
7 & 3 & 1 & $\sqrt{8}\left(3 \rho^{3}-2 \rho\right) \sin \theta$ & Primary $y$-coma \\
8 & 3 & 1 & $\sqrt{8}\left(3 \rho^{3}-2 \rho\right) \cos \theta$ & Primary $x$-coma \\
11 & 4 & 0 & $\sqrt{5}\left(6 \rho^{4}-6 \rho^{2}+1\right)$ & Primary Spherical \\
\hline
\end{tabular}

condition of Eq. (10). Thus, we obtain the vector function

$$
\vec{G}_{2}(\rho, \theta)=\frac{1}{3}\left(\rho^{2}-1\right) \cos \theta \hat{\rho}
$$

Now, we consider the vector function $\vec{G}_{2}(\rho, \theta)$ for which $G_{2 \rho}=0$ and write the solution in an abbreviated form,

$$
\frac{1}{\rho} \frac{\partial}{\partial \theta} G_{2 \theta}=\rho \cos \theta \quad, \quad G_{2 \theta}=\rho^{2} \sin \theta+f(\rho) \quad, \quad \vec{G}_{2}=\left[\rho^{2} \sin \theta+f(\rho)\right] \hat{\theta}
$$

If we combine the two solutions given by Eq. (18) and (19), we obtain another solution

$$
\vec{G}_{2}=a \frac{1}{3}\left(\rho^{2}-1\right) \cos \theta \hat{\rho}+b\left[\rho^{2} \sin \theta+f(\rho)\right] \hat{\theta} \quad, \quad a+b=1,
$$

where $a$ and $b$ can have any values subject to $a+b=1$. Evidently, we obtain an infinite number of vector functions for the wavefront tilt Zernike polynomial, as they satisfy the boundary condition. Similarly, we can construct vector functions for defocus and astigmatism, as outlined below. In each case, we obtain an infinite number of solutions.

\subsection{Defocus}

$$
\begin{aligned}
& Z_{4}(\rho, \theta)=2 \rho^{2}-1 \\
& G_{4 \rho}=0 \quad, \quad \frac{1}{\rho} \frac{\partial}{\partial \theta} G_{4 \theta}=2 \rho^{2}-1 \quad, \quad G_{4 \theta}=\left(2 \rho^{3}-\rho\right) \theta \quad, \quad \vec{G}_{4}=\left(2 \rho^{3}-\rho\right) \theta \hat{\theta},
\end{aligned}
$$




$$
\begin{aligned}
& G_{4 \theta}=0, \frac{1}{\rho} \frac{\partial}{\partial \rho}\left(\rho G_{4 \rho}\right)=2 \rho^{2}-1, \quad G_{4 \rho}=\frac{1}{2}\left(\rho^{3}-\rho\right), \quad \vec{G}_{4}=\frac{1}{2}\left(\rho^{3}-\rho\right) \hat{\rho}, \\
& \vec{G}_{4}=a \frac{1}{2}\left(\rho^{3}-\rho\right) \hat{\rho}+b\left(2 \rho^{3}-\rho\right) \theta \hat{\theta} \quad, \quad a+b=1 .
\end{aligned}
$$

\subsection{Astigmatism}

$$
\begin{aligned}
& Z_{6}(\rho, \theta)=\rho^{2} \cos 2 \theta \\
& G_{6 \rho}=0, \frac{1}{\rho} \frac{\partial}{\partial \theta} G_{6 \theta}=\rho^{2} \cos 2 \theta, G_{6 \theta}=\frac{1}{2} \rho^{3} \sin 2 \theta+f(\rho), \vec{G}_{6}=\left[\frac{1}{2} \rho^{3} \sin 2 \theta+f(\rho)\right] \hat{\theta}, \\
& G_{6 \theta}=0, \frac{1}{\rho} \frac{\partial}{\partial \rho}\left(\rho G_{6 \rho}\right)=\rho^{2} \cos 2 \theta, G_{6 \rho}=\frac{1}{4}\left(\rho^{3}-1\right) \cos 2 \theta, \vec{G}_{6}=\frac{1}{4}\left(\rho^{3}-1\right) \cos 2 \theta \hat{\rho}, \\
& \vec{G}_{6}=a \frac{1}{4}\left(\rho^{3}-1\right) \cos 2 \theta \hat{\rho}+b \frac{1}{2} \rho^{3} \sin 2 \theta \hat{\theta} \quad, \quad a+b=1 .
\end{aligned}
$$

Similarly, we can find vector function(s) for each $Z_{j}(\rho, \theta)$.

\section{NOISE PROPAGATION}

Given an infinite number of vector functions that yield the Zernike coefficients from the wavefront slope data, how do we decide which of them to use? Are some vector functions better than others? In what sense? Is there a systematic way to choose the right set of vector functions? Let us consider the effect of noise in the slope data on the

Zernike coefficients We show that when the data are noisy, which inevitably they are, irrotational vector functions (i.e., those whose curls are zero) [5-7] propagate the least noise from the data to the coefficients. This derivation is independent of the shape of the pupil, e.g., it is applicable to annular pupils.

Consider an irrotational vector function $\boldsymbol{G}_{j}(\boldsymbol{r})$ obtained as the gradient of a scalar function $U_{j}(\boldsymbol{r})$,

$$
\boldsymbol{G}_{j}(\boldsymbol{r})=\nabla U_{j}(\boldsymbol{r})
$$

with the boundary condition

$$
\boldsymbol{G}_{j}(|\boldsymbol{r}|=1)=0 .
$$

If $\boldsymbol{n}(\boldsymbol{r})$ is the uncorrelated additive random noise with zero mean and covariance

$$
\left\langle\boldsymbol{n}(\boldsymbol{r}) \cdot \boldsymbol{n}\left(\boldsymbol{r}^{\prime}\right)\right\rangle=\sigma_{n}^{2} \delta\left(\boldsymbol{r}-\boldsymbol{r}^{\prime}\right),
$$

then the estimated coefficient may be written

$$
\tilde{a}_{j}=\frac{1}{\pi} \int_{0}^{1} \int_{0}^{2 \pi} \boldsymbol{G}_{j}(\boldsymbol{r}) \cdot[\nabla W(\boldsymbol{r})+\boldsymbol{n}(\boldsymbol{r})] d^{2} \boldsymbol{r} .
$$

The error in the coefficient values is given by 


$$
\tilde{a}_{j}-a_{j}=\frac{1}{\pi} \int_{0}^{1} \int_{0}^{2 \pi} \boldsymbol{G}_{j}(\boldsymbol{r}) \cdot \boldsymbol{n}(\boldsymbol{r}) d^{2} \boldsymbol{r}
$$

The corresponding error variance is given by

$$
\begin{aligned}
\sigma_{j}^{2} & =\left\langle\left(\tilde{a}_{j}-a_{j}\right)^{2}\right\rangle \\
& =\sigma_{n}^{2} \frac{1}{\pi^{2}} \int_{0}^{1} \int_{0}^{2 \pi}\left|\boldsymbol{G}_{j}(\boldsymbol{r})\right|^{2} d^{2} \boldsymbol{r} .
\end{aligned}
$$

Thus, the noise propagator may be written

$$
N_{p}\left(G_{j}\right)=\frac{1}{\pi^{2}} \int_{0}^{1} \int_{0}^{2 \pi}\left|G_{j}(r)\right|^{2} d^{2} r
$$

The error variance depends only on the chosen vector function $\boldsymbol{G}_{j}(\boldsymbol{r})$.

To show that the noise propagator given by Eq. (8) imparts minimum noise from the slope data to the coefficients, we consider another vector function $\boldsymbol{F}_{j}(\boldsymbol{r})$ such that

$$
\boldsymbol{F}_{j}(\boldsymbol{r})=\boldsymbol{G}_{j}(\boldsymbol{r})+\boldsymbol{A}_{j}(\boldsymbol{r}),
$$

where $\boldsymbol{A}_{j}(\boldsymbol{r})$ is a solenoidal vector function, i.e., whose divergence is zero,

$$
\nabla \cdot \boldsymbol{A}_{j}(\boldsymbol{r})=0
$$

and obeys the condition

$$
\boldsymbol{A}_{j}(|\boldsymbol{r}|=1)=0
$$

The noise propagator associated with the vector function $\boldsymbol{F}_{j}(\boldsymbol{r})$ is given by

$$
\begin{aligned}
N_{p}\left(F_{j}\right) & =\frac{1}{\pi^{2}} \int_{0}^{1} \int_{0}^{2 \pi}\left|F_{j}(r)\right|^{2} d^{2} r \\
& =N_{p}\left(G_{j}\right)+N_{p}\left(A_{j}\right)+2 \frac{1}{\pi^{2}} \int_{0}^{1} \int_{0}^{2 \pi} G_{j}(r) \cdot A_{j}(r) d^{2} r .
\end{aligned}
$$

From the divergence theorem

$$
\nabla \cdot\left(U_{j} \boldsymbol{A}_{j}\right)=U_{j} \nabla \cdot \boldsymbol{A}_{j}+\boldsymbol{A}_{j} \cdot\left(\nabla U_{j}\right)=\boldsymbol{A}_{j} \cdot \boldsymbol{G}_{j},
$$

where we have used Eqs. (23) and (31). Integration both sides of Eq. (13), we may write

$$
\begin{aligned}
\int_{0}^{1} \int_{0}^{2 \pi} \boldsymbol{G}_{j}(\boldsymbol{r}) \cdot \boldsymbol{A}_{j}(\boldsymbol{r}) d^{2} \boldsymbol{r} & =\int_{0}^{1} \int_{0}^{2 \pi} \nabla \cdot\left(U_{j} \boldsymbol{A}_{j}\right) d^{2} \boldsymbol{r} \\
& =\oint_{1} U(|\boldsymbol{r}|=\boldsymbol{1}) \boldsymbol{A}_{j}(|\boldsymbol{r}|=\mathbf{1}) \cdot d \boldsymbol{l} \\
& =0,
\end{aligned}
$$


where we have used Eq. (32) in the last step. Therefore, Eq. (33) reduces to

$$
N_{p}\left(\boldsymbol{F}_{j}\right)=N_{p}\left(\boldsymbol{G}_{j}\right)+N_{p}\left(\boldsymbol{A}_{j}\right)
$$

Since by definition, $N_{p}\left(\boldsymbol{A}_{j}\right) \geq 0$, it follows from Eq. (36) that $N_{p}\left(\boldsymbol{F}_{j}\right) \geq N_{p}\left(\boldsymbol{G}_{j}\right)$, or $N_{p}\left(\boldsymbol{G}_{j}\right)$ is minimum.

\section{IRROTATIONAL VECTOR FUNCTIONS}

Now, we derive vector functions that are irrotational, i.e., whose curls are zero, so that they propagate minimum noise from the slope data to the aberration coefficients [5-7]. This property is satisfied if they are obtained as the gradient of a scalar function $U_{j}(\rho, \theta)$, i.e., if

$$
\vec{G}_{j}(\rho, \theta)=\nabla U_{j}(\rho, \theta) .
$$

Substituting Eq. (37) into Eq. (9), we obtain a Poisson equation

$$
\nabla^{2} U_{j}(\rho, \theta)=-Z_{j}(\rho, \theta)
$$

that we must solve subject to the boundary condition of Eq. (10). In polar coordinates, Eq.(38) can be written

$$
\frac{1}{\rho} \frac{\partial}{\partial \rho}\left[\rho \frac{\partial}{\partial \rho} U_{j}(\rho, \theta)\right]+\frac{1}{\rho^{2}} \frac{\partial^{2}}{\partial \theta^{2}}\left[U_{j}(\rho, \theta)\right]=-Z_{j}(\rho, \theta),
$$

Substituting for the Zernike polynomials, as

$$
\begin{aligned}
& Z_{j}(\rho, \theta)=\sqrt{n+1} R_{n}^{0}(\rho), m=0, \\
& {\left[\begin{array}{c}
Z_{\text {even } j}(\rho, \theta) \\
Z_{\text {odd } j}(\rho, \theta)
\end{array}\right]=\sqrt{2(n+1)} R_{n}^{m}(\rho)\left(\begin{array}{c}
\cos m \theta \\
\sin m \theta
\end{array}\right), m \neq 0,}
\end{aligned}
$$

it can be shown [7] that the vector functions are polynomials given by:

$$
\begin{aligned}
& \vec{G}_{j}(\rho, \theta)=G_{j}(\rho) \hat{\rho}=-\sqrt{(n+1)} \sum_{s=0}^{n / 2} \frac{E_{n}^{0}(s)}{b(s)} \rho^{b-1}(\cos \theta \hat{i}+\sin \theta \hat{j}), m=0, \\
& \vec{G}_{\text {even } j}(\rho, \theta)=-\sqrt{2(n+1)} \sum_{s=0}^{(n-m) / 2} \frac{E_{n}^{m}(s)}{b^{2}-m^{2}}(L \hat{i}+M \hat{j}), \\
& \vec{G}_{\text {odd } j}(\rho, \theta)=-\sqrt{2(n+1)} \sum_{s=0}^{(n-m) / 2} \frac{E_{n}^{m}(s)}{b^{2}-m^{2}}\left(L^{\prime} \hat{i}+M \hat{j}\right),
\end{aligned}
$$

where

$$
\begin{aligned}
& L(s)=\frac{b-m}{2} \rho^{b-1} \cos (m+1) \theta+\left(\frac{b+m}{2} \rho^{b-1}-b \rho^{m-1}\right) \cos (m-1) \theta \\
& M(s)=\frac{b-m}{2} \rho^{b-1} \sin (m+1) \theta-\left(\frac{b+m}{2} \rho^{b-1}-b \rho^{m-1}\right) \sin (m-1) \theta,
\end{aligned}
$$




$$
\begin{aligned}
& L^{\prime}(s)=\frac{b-m}{2} \rho^{b-1} \sin (m+1) \theta+\left(\frac{b+m}{2} \rho^{b-1}-b \rho^{m-1}\right) \sin (m-1) \theta \\
& M^{\prime}(s)=-\frac{b-m}{2} \rho^{b-1} \cos (m+1) \theta+\left(\frac{b+m}{2} \rho^{b-1}-b \rho^{m-1}\right) \cos (m-1) \theta \\
& b(s)=n-2 s+2
\end{aligned}
$$

and

$$
E_{n}^{m}(s)=\frac{(-1)^{s}(n-s) !}{s !\left(\frac{n+m}{2}-s\right) !\left(\frac{n-m}{2}-s\right) !} .
$$

The vector polynomials for primary aberrations are given in Table 2 .

\section{VECTOR FUNCTIONS FOR AN ANNULAR WAVEFRONT}

We outline the differences of the annular vector functions [8] from the vector polynomials for a circular pupil [7]. The radial integrations for an annular pupil are from $\epsilon$ to 1 , where $\epsilon$ is its obscuration ratio (instead of from 0 to 1, as for a circular pupil), and we use the annular polynomials [1] in Eq. (9) in place of the Zernike polynomials. The boundary conditions are based on two line integrals, one at $\rho=\epsilon$ and the other at $\rho=1$. The procedure to obtain the annular vector function is otherwise very similar to that for a circular wavefront and relatively straightforward, though their mathematical expressions are long, requiring careful bookkeeping.

Table 2. Vector polynomials $\vec{G}_{j}(\rho, \theta)$ for primary aberrations for a circular wavefront

\begin{tabular}{llll}
\hline$j$ & $n$ & $m$ & $-\vec{G}_{j}(\rho, \theta)$ \\
\hline 2 & 1 & 1 & $(1 / 4)\left[\left(\rho^{2} \cos 2 \theta+2 \rho^{2}-3\right) i+\rho^{2} \sin 2 \theta \hat{j}\right]$ \\
3 & 1 & 1 & $(1 / 4)\left[\rho^{2} \sin 2 \theta \hat{i}+\left(-\rho^{2} \cos 2 \theta+2 \rho^{2}-3\right) \hat{j}\right]$ \\
4 & 2 & 0 & $(\sqrt{3} / 2)\left(\rho^{3}-\rho\right)(\cos \theta \hat{i}+\sin \theta \hat{j})$ \\
5 & 2 & 2 & $(1 / \sqrt{24})\left\{\left[\rho^{3} \sin 3 \theta+\left(3 \rho^{3}-4 \rho\right) \sin \theta\right] \hat{i}+\left[-\rho^{3} \cos 3 \theta+\left(3 \rho^{3}-4 \rho\right) \cos \theta\right] \hat{j}\right\}$ \\
6 & 2 & 2 & $(1 / \sqrt{24})\left\{\left[\rho^{3} \cos 3 \theta+\left(3 \rho^{3}-4 \rho\right) \cos \theta\right] \hat{i}+\left[\rho^{3} \sin 3 \theta-\left(3 \rho^{3}-4 \rho\right) \sin \theta\right] \hat{j}\right\}$ \\
7 & 3 & 1 & $(1 / \sqrt{8})\left\{2\left(\rho^{4}-\rho^{2}\right) \sin 2 \theta \hat{i}+\left[-2\left(\rho^{4}-\rho^{2}\right) \cos 2 \theta+3 \rho^{4}-4 \rho^{2}+1\right] \hat{j}\right\}$ \\
8 & 3 & 1 & $(1 / \sqrt{8})\left\{\left[2\left(\rho^{4}-\rho^{2}\right) \cos 2 \theta+3 \rho^{4}-4 \rho^{2}+1\right] \hat{i}+2\left(\rho^{4}-\rho^{2}\right) \sin 2 \theta \hat{j}\right\}$ \\
11 & 4 & 0 & $(\sqrt{5} / 2)\left(2 \rho^{5}-3 \rho^{3}+\rho\right)(\cos \theta \hat{i}+\sin \theta \hat{j})$ \\
\hline
\end{tabular}


There is no generating function for the radial annular polynomials, except when $m=0, m=n$, or $m=n-2$. Hence, no general expression for the vector functions can be obtained. However, only 12 of the first 45 vector functions for which $n \leq 8$ don't belong to the $m=0, m=n$, or $m=n-2$ category. They are for $(n, m)=(5,1)$, $(6,2),(7,1),(7,3),(8,2)$ and $(8,4)$. As expected, the annular vector functions reduce to the corresponding vector polynomials for a circular wavefront as $\epsilon \rightarrow 0$.

\section{DISCUSSION AND CONCLUSIONS}

We have shown that an infinite number of vector functions exist whose inner products with the slope data of a circular wavefront yield the Zernike aberration coefficients. However, the irrotational functions transport minimum noise from the slope data to the coefficients. The vector functions obtained as the gradient of a scalar function are irrotational. The vector functions for a circular wavefront are polynomials [7]. Both the Zernike and vector polynomials are given in Tables 1 and 2 for primary aberrations. As expected, the degree of a vector polynomial is one higher than that of a corresponding Zernike polynomial. These vector polynomials can be used in Eq. (3) to obtain the Zernike coefficients directly from the wavefront slope data. Unlike the Zernike polynomials, the vector polynomials are not orthogonal to each other.

A comprehensive derivation of the irrotational vector polynomials for a circular wavefront is given in a separate paper [7]. A similar derivation of the irrotational vector functions for an annular wavefront is also given in another paper [8]. These vector functions are not polynomials.

\section{REFERENCES}

[1] V. N. Mahajan, Optical Imaging and Aberrations, Part III: Wavefront Analysis, SPIE Press (2013).

[2] J. Braat, "Polynomial expansion of severely aberrated wavefronts," J. Opt. Soc. Am. A 4, 643-650 (1987).

[3] C. Zhao and J. H. Burge, "Orthonormal vector polynomials in a unit circle, Part I: basis set derived from gradients of Zernike polynomials," Opt. Express 15,18014-18024 (2007).

[4] A. Gavrielides, "Vector polynomials orthogonal to the gradient of Zernike polynomials," Opt. Lett. 7, 526-528 (1982).

[5] C. Solomon, S. Ríos, E. Acosta, and S. Bará, "Modal wavefront projectors of minimum error norm," Opt. Commun. 155, 252-254 (1998).

[6] C. Solomon, G. C. Loos, and S. Ríos, "Variational solution for modal wavefront projection functions of minimum error norm," J. Opt. Soc. Am. A 18, 1519-1522 (2001).

[7] V. N. Mahajan and Eva Acosta, "Vector polynomials for direct analysis of circular wavefront slope data," J. Opt. Soc. Am. A. 34, 1908-1913 (2017).

[8] E. Acosta and V. N. Mahajan, "Vector functions for direct analysis of annular wavefront slope data," to be published

[9] I. S. Gradshteyn and I. M. Ryzhik, "Tables of Integrals, Series, and Products," Chapter 10, Academic Press, New York (1980).

[10] E. Acosta, S. Bará, M. A. Rama, and S Ríos, "Determination of phase mode components in terms of local wave-front slopes: an analytical approach,” Opt. Lett. 20, 1083-1085 (1995). 